\title{
Focused Interest Group on Microanalytical Standards (FIGMAS): Assessing the Quality, Availability and Need for Standards in the Microanalytical Community
}

\author{
Julien M. Allaz ${ }^{1}$, Owen K. Neill ${ }^{2}$ and Anette von der Handt ${ }^{3}$ \\ 1. University of Colorado Boulder, Department of Geological Sciences, Boulder (CO), USA \\ 2. Washington State University, Peter Hooper GeoAnalytical Laboratory, School of the Environment, \\ Pullman (WA), USA \\ 3. University of Minnesota Twin Cities, Department of Earth Sciences, Minneapolis (MN), USA
}

It has been recognized over the past years that different electron microprobe and scanning electron microprobe laboratories use different sets of standards or reference materials for quantitative analysis. Unfortunately, some of these standards either have become unavailable (e.g., some natural minerals from the Smithsonian Institution collection) or are only available to a restricted group of people (e.g., internal reference materials). Other synthetic materials are also available commercially or provided by other institutions and research centers. However, they sometimes lack either broad availability or acceptable characterization (e.g., NIST glasses, Corning glasses, Drake \& Weill REE-glasses... [1,2]). Another important problem for the community is a clear assessment of standard quality (the "Good", "Bad" and "Ugly" of Carpenter [3]): "good" homogeneous standards with accurate compositional information and without impurities or inclusions are rare, whereas "bad" standards, which lack good characterization, are more common. Individual lab managers do commonly examine their own standard collections to re-evaluate compositional homogeneity and test the accuracy of published compositions. Standards are also frequently re-analyzed at individual labs using various techniques, and therefore multiple accepted compositions for individual standards may exist.

This information about which standards are available, how to obtain new standards, and which existing standards are "Good", "Bad", or "Ugly" (to use Carpenter's [3] terminology) is incredibly valuable to the microprobe community, but such information is only rarely disseminated in any systematic way. In fact, the most complete published review of common microprobe standards is now 30 years old [4]. More recently, other researchers have reviewed limited sets of natural and synthetic Smithsonian microbeam standards to check for their homogeneity and the presence of mineral inclusions or impurities. This includes studies on the composition, quality, homogeneity and presence of impurity in many of these standards [5,6,7,8], a study on the presence of $\mathrm{Pb}$ in the synthetic REE-phosphate [9], an evaluation of the micro- to nano-scale impurities in Kakanui Hornblende [10], and a review of the quality of several pyroxene standards [11].

The community would benefit greatly from an organized and widely-available repository of information about standards and reference materials (composition, standard quality, availability, source, etc.). To address these issues, the Focused Interest Group for MicroAnalytical Standards (FIGMAS*) aims to promote and facilitate the creation of a community-wide standard materials collection that supports consistency and inter-laboratory comparison. Part of this effort will be creating an online database of the currently available standard and reference materials used in electron microprobe laboratories. This database will be used to assess the need of additional reference materials for quantitative electron microprobe analyses by energy- and wavelength-dispersive X-ray spectroscopy. In addition, the FIGMAS will assess the needs of the microprobe community regarding collection and production of new standard or reference materials. The FIGMAS will also collect known procedures for synthesizing 
or sintering a homogeneous standard material (glass or mineral), and, where possible, make these procedures available to the greater microbeam analysis community. If necessary, the FIGMAS will also serve to develop new such procedures. In the future, this database could be extended and crossreferenced to other reference material database already available for other analytical techniques (e.g., GEOREM for laser ablation ICP-MS, SHRIMP or SIMS [12]). The preparation of some round robin test of commonly used standard is a possibility.

The FIGMAS seeks to determine the availability and quality of each standard and reference material used and to define them as Standard Reference Material (SRM), Reference Material (RM) or Certified Reference Material (CRM) following the definition from the National Institute of Standards and Technology (NIST). The group will particularly focus on criteria such as (a) the quantity available and average particle size, (b) the compositional homogeneity, (c) pervasiveness of impurities or inclusions, (d) the resistance to beam damage and to high vacuum, (e) the non-soluble aspect of the material in most eluent used for polishing and cleaning, (f) the long term stability (potential for oxidation, hydration, or change in crystal structure, etc.), and ( $\mathrm{g}$ ) the availability and quality of quantitative analyses for each of the material's constituent elements, including the analytical method used and the availability of a certificate from a trusted source (e.g., NIST or a similar recognized institution).

The results of the FIGMAS will be made available on a website that the microbeam community can use as reference. This database will be accompanied by publications in peer-reviewed scientific journals. The analytical community will be given access to the database to facilitate the addition of new reference material data. FIGMAS committee members will be responsible for reviewing any entry made by a member of the community to validate its provenance, delete inappropriate or spurious entries, consolidate duplicates, and ensure completeness of the record. With the help of the wider microbeam community, we hope that this database will constantly evolve to widely distribute this valuable information, enhance inter-laboratory comparability, and permit some poor "bad" standards to, one day, join the "good" standards realm.

* The request for the creation of this FIG is pending. Researchers and laboratory managers interested to join this group are encouraged to contact one of the authors (julien.allaz@colorado.edu, owen.neill@wsu.edu,oravdhandt@umn.edu).

References:

[1] P.K. Carpenter et al., J. Res. Natl. Inst. Stand. Technol. 107 (2002), 703-718.

[2] M.J. Drake and D.F. Weill, Chemical Geology 10 (1972), 179-181.

[3] P.K. Carpenter, Microscopy \& Microanalysis meeting 14 (2008), 530-531.

[4] J.S. Huebner and M.E. Woodruff, US Geological Survey Open File Report 85-718 (1985), 237 pp.

[5] E. Jarosewich, J.A. Nelen and J.A. Norberg, Geostandard newsletter 4 (1980), 43-47.

[6] E. Jarosewich, J. Res. Natl. Inst. Stand. Technol. 107 (2002), 681-685.

[7] T.R. Rose, Microscopy \& Microanalysis meeting 14 (2008), 528-529.

[8] http://mineralsciences.si.edu/facilities/standards.htm

[9] J.J. Donovan et al., The Canadian Mineralogist 41 (2003), 221-232.

[10] E.P. Vicenzi and T. Rose, Microscopy \& Microanalysis meeting 14 (2008), 522-523.

[11] J. Fournelle, AGU Fall meeting (2012), V23C-2827.

[12] http://georem.mpch-mainz.gwdg.de 